\title{
Changes in Optical Coherence Tomography Findings in Acute Zonal Occult Outer Retinopathy
}

\author{
Shinji Makino Hironobu Tampo \\ Department of Ophthalmology, Jichi Medical University, Shimotsuke, Japan
}

\section{Key Words}

Acute zonal occult outer retinopathy - Optical coherence tomography · Photoreceptor inner segment/outer segment junction · Cone outer segment tip · Multifocal electroretinogram

\begin{abstract}
Purpose: To report a case of acute zonal occult outer retinopathy (AZOOR) in a 39-year-old woman. Methods: Images were obtained with fundus photography and optical coherence tomography (OCT). A multifocal electroretinogram (mfERG) was also obtained to evaluate retinal function. Results: The patient's right visual acuity was 0.8. Fundus photographs showed no specific abnormal findings. OCT showed attenuation of the photoreceptor inner segment/outer segment (IS/OS) line and the cone outer segment tip (COST) line in the right eye. The mfERG showed corresponding amplitude reductions. One month after the initial visit, her right visual acuity improved to 1.2. There was partial recovery of the IS/OS line; however, the COST line was still absent, and there was no apparent improvement in the mfERG responses. Conclusions: Our findings showed a discrepancy between the microstructural findings and visual function during recovery of AZOOR.

(c) 2013 S. Karger AG, Basel
\end{abstract}

\section{Introduction}

Acute zonal occult outer retinopathy (AZOOR) is characterized by an acute zonal loss of outer retinal function involving one or both eyes. AZOOR occurs predominantly in young women. Initially, minimal or no fundus changes occur, but enlarged blind spots, abnormalities on electroretinograms (ERGs) and permanent visual field loss often occur in this slowly progressing form of retinal pigment epithelium degeneration $[1,2]$. Optical coherence tomography (OCT) images from eyes with AZOOR show a loss or irregularity of the 
Makino et al.: Changes in Optical Coherence Tomography Findings in Acute Zonal Occult Outer Retinopathy

photoreceptor inner segment/outer segment (IS/OS) line in areas corresponding to reduced multifocal ERG (mfERG) responses and visual field defects [3-15]. Recently, the cone outer segment tip (COST) line has been recognized as a thin, highly reflective line located between the IS/OS junction and the retinal pigment epithelium [16]. To our knowledge, there are few reports in the literature describing the abnormalities of the COST line in AZOOR [8]. However, there are several detailed reports about AZOOR patients who showed subjective and objective improvements in visual function [4, 8-15]. Here, we report an AZOOR patient with partial restoration of the IS/OS line.

\section{Case Report}

A 39-year-old Japanese woman presented with a complaint of photopsias and central scotoma in her right eye. Best corrected visual acuity (BCVA) was 0.7 and $1.2 \mathrm{in} \mathrm{her} \mathrm{right} \mathrm{and}$ left eyes, respectively. Intraocular pressure was $16 \mathrm{~mm} \mathrm{Hg}$ in each eye. The patient had no specific past medical history. No inflammatory cells were observed in the anterior segment or vitreous of either eye, and there were no specific abnormal findings during a fundus examination (fig. 1a, f).

OCT (RS-3000; Nidek, Japan) showed that while there was attenuation of the IS/OS line throughout the entire region of her right eye and the COST line (fig. 1b, c), no specific abnormalities were detected in her left eye (fig. 1g, h). mfERG (LE-4100; Tomey, Japan) findings showed markedly decreased amplitudes in the right eye (fig. 1d, e) and no abnormalities in the left eye (fig. 1i, j). The patient was followed without any treatment. BCVA improved to 0.9 in the right eye and OCT revealed there was no recovery of the IS/OS at the foveal area at 1 month after the initial visit (fig. 2a). After 2 months, BCVA improved to 1.2 in the right eye and OCT showed a partial restoration of the IS/OS line (fig. 2b); however, the COST line was still absent, and she had no change in mfERG findings. At 4 months, reappearance of the IS/OS line was detected by OCT (fig. 2c). However, the COST line was still absent, and she showed no improvement in the mfERG findings. Fluorescein angiography and indocyanine green angiography were not available in this case.

\section{Discussion}

Our case showed partial restoration of the IS/OS line defect, which was not associated with functional recovery. Our OCT findings were similar to those previously reported for other AZOOR patients [3-15]. It has been reported that the natural course of AZOOR is varied [1, 2]. Gass et al. [2] performed a long-term follow-up study of the clinical course of AZOOR and reported that visual field loss stabilized within 6 months in 37 (72\%) of 51 patients, progressed stepwise in 2 (4\%) patients and partly improved in $12(24 \%)$ other patients.

There have been several detailed reports about AZOOR patients who showed morphological and functional improvements in their visual function [4, 8-15]. Spaide et al. [4] reported restoration of the IS/OS line in the improved areas of the visual field, but electrophysiological alterations of these patients were not demonstrated in the report. Chai et al. [14] reported a case with restoration of the IS/OS line and mild improvement of the mfERG. Yasuda et al. [15] reported a case with mild improvement of the mfERG, but morphological changes were not demonstrated in the report. 
Makino et al:: Changes in Optical Coherence Tomography Findings in Acute Zonal Occult Outer Retinopathy

Fujiwara et al. [17] described IS/OS boundary abnormalities in all 19 eyes studied. Fourteen (74\%) eyes showed some absence of the IS/OS boundary, and 5 (26\%) eyes showed attenuation of the IS/OS boundary on OCT scans. Srinivasan et al. [16] reported that the highly reflective line between the IS/OS junction and the retinal pigment epithelium was the COST line, and it was visible because of the different lengths of the cone and rod outer segments. A correlation was found between the site of the visual impairments and the loss of the foveal COST line in several retinal diseases including macular holes, occult macular dystrophy and the AZOOR complex [18]. All of these reports focused on the microstructures and reported that the integrity of the microstructures were correlated with the BCVA; i.e., absence of the IS/OS line and/or the COST lines were present in eyes with decreased BCVA, and their intactness coexisted with an improved BCVA. In addition, a significant correlation was present between these morphological changes and the BCVA. However, to our knowledge, there have been few reports in the literature describing the abnormalities of the COST line in AZOOR [8]. So et al. [8] described a discrepancy between the microstructural findings and visual function; i.e., good BCVA, normal visual fields and normal-size mfERGs in spite of an absence of the COST line. They also described that an absence of the COST line was observed even after recovery of the cone function detected by perimetry and an mfERG. These findings suggest that the COST line is the most sensitive parameter for the pathology of the retina. In addition, Mkrtchyan et al. [19] demonstrated changes in the outer retinal structure in 4 AZOOR patients using adaptive optics scanning laser ophthalmoscopy. Adaptive optics scanning laser ophthalmoscopy showed reduced cone reflectivity, absence of cones in the region of scotoma and abnormal cone coverage based on direct visualization of the photoreceptors in living retina.

In conclusion, our findings showed a discrepancy between the microstructural findings and visual function in the recovery of AZOOR. Our findings were based on a single case of AZOOR and further studies with additional cases are necessary.

\section{Disclosure Statement}

The authors have no conflicts of interest to disclose.

\section{References}

1 Monson DM, Smith JR: Acute zonal occult outer retinopathy. Surv Ophthalmol 2011;56:23-35.

$\longrightarrow 2$ Gass JD, Agarwal A, Scott IU: Acute zonal occult outer retinopathy: a long-term follow-up study. Am J Ophthalmol 2002;134:329-339.

-3 Li D, Kishi S: Loss of photoreceptor outer segment in acute zonal occult outer retinopathy. Arch Ophthalmol 2007;125:1194-1200.

4 Spaide RF, Koizumi H, Freund KB: Photoreceptor outer segment abnormalities as a cause of blind spot enlargement in acute zonal occult outer retinopathy-complex diseases. Am J Ophthalmol 2008;146:111120.

-5 Takai Y, Ishiko S, Kagokawa H, Fukui K, Takahashi A, Yoshida A: Morphological study of acute zonal occult outer retinopathy (AZOOR) by multiplanar optical coherence tomography. Acta Ophthalmol 2009;87:408418.

6 Ohta K, Sato A, Fukui E: Spectral domain optical coherence tomographic findings at convalescent stage of acute zonal occult outer retinopathy. Clin Ophthalmol 2009;3:423-428.

7 Makino S, Tanaka Y, Tampo H: An optical coherence tomography and fundus autofluorescence imaging study of peripapillary acute zonal occult outer retinopathy. Case Rep Ophthalmol 2013;4:11-16.

-8 So K, Shinoda K, Matsumoto CS, Satofuka S, Imamura Y, Mizota A: Focal functional and microstructural changes of photoreceptors in eyes with acute zonal occult outer retinopathy. Case Rep Ophthalmol 2011;2:307-313. 
Makino et al:: Changes in Optical Coherence Tomography Findings in Acute Zonal Occult Outer Retinopathy

-9 Kitakawa T, Hayashi T, Takashina H, Mitooka K, Gekka T, Tsuneoka H: Improvement of central visual function following steroid pulse therapy in acute zonal occult outer retinopathy. Doc Ophthalmol 2012;124:249-254.

10 Mizoguchi T, Tanikawa A, Horiguchi M: The changes in optical coherence tomography (OCT) findings in acute zonal occult outer retinopathy. Folia Jap Ophthalmol Clin 2009;2:735-738.

11 Hara K, Terada Y, Akimoto E, Shibata K: A case of acute zonal occult outer retinopathy with rapid improvement. J Eye 2012;29:1426-1428.

12 Matsuda H, Sakai T, Kouzaki K, Nakano T, Shikisima K, Tsuneoka H: A bilateral case of acute zonal occult outer retinopathy (AZOOR) with improved rod dysfunction. Jpn J Clin Ophthalmol 2010;64:733-738.

13 Ohshima A, Hasegawa S, Abe H, Takagi M, Takada R, Wakai M, Ishii S: A case of acute zonal occult outer retinopathy identified by multifocal electroretinography changes. Folia Ophthalmol Jpn 1997;48:829-832.

14 Chai Y, Yamazaki H, Fujinami K, Tsunoda K, Yamamoto S: Case of acute zonal occult outer retinopathy with abnormal pattern visual evoked potentials. Clin Ophthalmol 2011;5:1235-1241.

15 Yasuda K, Shimura M, Noro M, Nakazawa M, Tamai K: Clinical course of acute zonal occult outer retinopathy in visual field and multifocal electroretinogram. Br J Ophthalmol 1999;83:1089-1090.

-16 Srinivasan VJ, Monson BK, Wojtkowski M, Bilonock RA, Gorczynska I, Chen R, Duker JS, Schuman JS, Fujimoto JG: Characterization of outer retinal morphology with highspeed, ultrahigh-resolution optical coherence tomography. Invest Ophthalmol Vis Sci 2008;49:1571-1579.

17 Fujiwara T, Imamura Y, Giovinazzo VJ, Spaide RF: Fundus autofluorescence and optical coherence tomographic findings in acute zonal occult outer retinopathy. Retina 2010;30:1206-1216.

18 Sugahara M, Shinoda K, Matsumoto SC, Satofuka S, Hanazono G, Imamura Y, Mizota A: Outer retinal microstructure in a case of acute idiopathic blind spot enlargement syndrome. Clin Ophthalmol 2011;2:116-122.

19 Mkrtchyan M, Lujan BJ, Merino D, Thirkill CE, Roorda A, Duncan JL: Outer retinal structure in patients with acute zonal occult outer retinopathy. Am J Ophthalmol 2012;153:757-768. 


\section{Case Reports in \\ Ophthalmology}

\begin{tabular}{l|l}
\hline Case Rep Ophthalmol 2013;4:99-104 & \\
\hline DOI: 10.1159/000355108 & $\begin{array}{l}\text { @ 2013 S. Karger AG, Basel } \\
\text { www.karger.com/cop }\end{array}$ \\
\hline
\end{tabular}

Makino et al:: Changes in Optical Coherence Tomography Findings in Acute Zonal Occult Outer Retinopathy
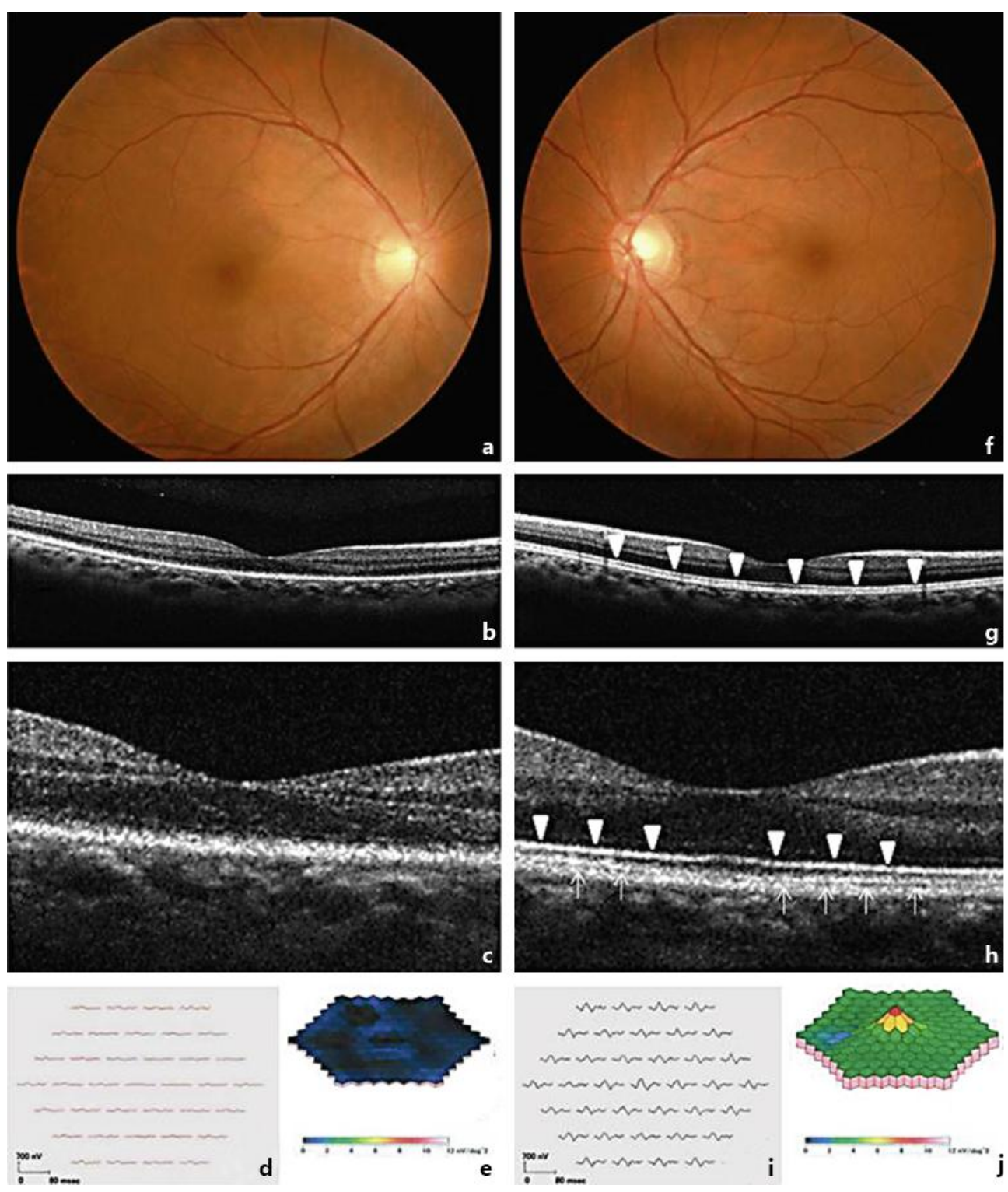

Fig. 1. Findings in a 39-year-old woman with AZOOR in the right (a-e) and left $(\mathbf{f}-\mathbf{j})$ eyes. Fundus photographs show no specific abnormalities (a, f). OCT horizontal scan (b) and high-magnification OCT findings (c) show an absent photoreceptor IS/OS line and the COST line within AZOOR lesions (in the macula area) in the right eye. In the left eye, the IS/OS line and the COST line were detected $(\mathbf{g}, \mathbf{h})$. mfERG response arrays $(\mathbf{d}, \mathbf{i})$ and 3-dimensional plots $(\mathbf{e}, \mathbf{j})$ showed reduced responses in the right eye. Arrowheads indicate the IS/OS line. Arrows indicate the COST line. 
Makino et al.: Changes in Optical Coherence Tomography Findings in Acute Zonal Occult Outer Retinopathy
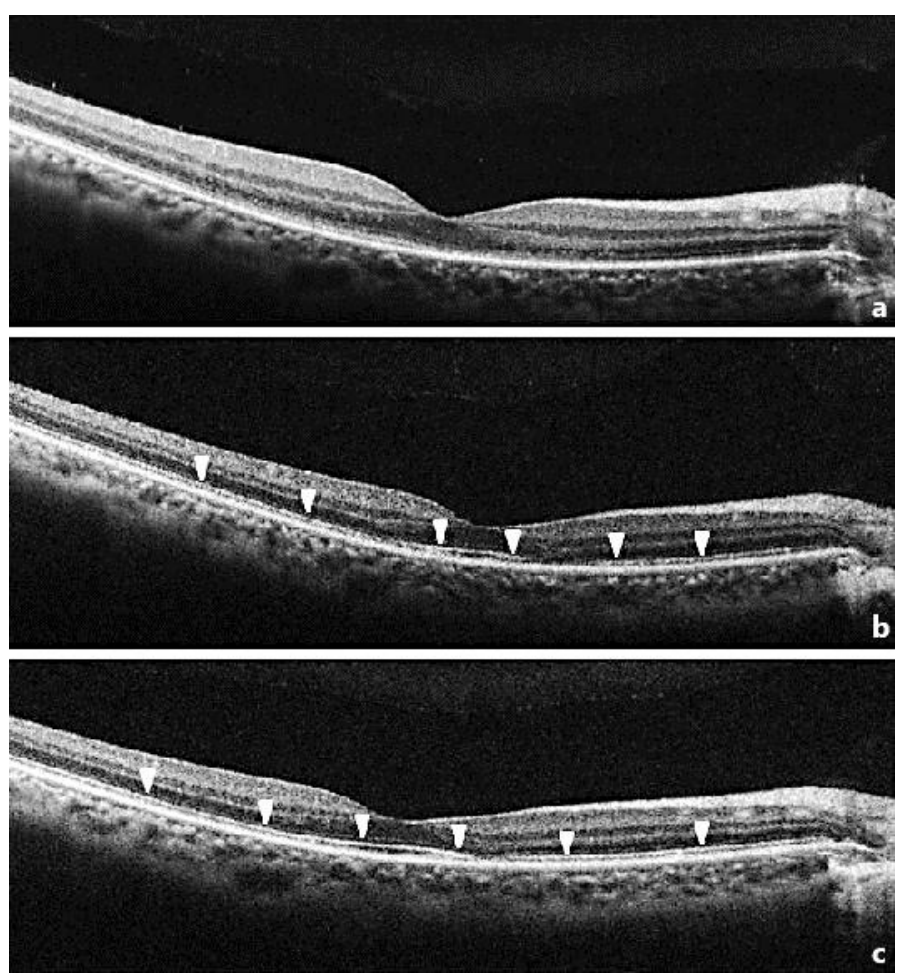

Fig. 2. Changes in high-magnification OCT findings in a 39-year-old woman with AZOOR in the right eye (a-c). At the initial visit, OCT showed attenuation of the IS/OS line and the COST line (a). After 2 months, OCT showed a partial restoration of the IS/OS line (b); however, the COST line was still absent. At 4 months, reappearance of the IS/OS line was detected by OCT (c); however, the COST line was still absent. Arrowheads indicate the IS/OS line. 